\title{
Calidad sanitaria e inocuidad alimentaria de cárnicos, según atributos físicos, químicos y microbiológicos
} utilizando una técnica multivariada

Sanitary quality and food safety of meat, according to physical, chemical and microbiological attributes using a multivariate technique

Jhony A. Gonzales-Malca ${ }^{1^{*}}\left(\mathbb{D}\right.$, María Santos Abanto-López ${ }^{1}$ (D)

\section{RESUMEN}

La carne, como fuente de enfermedades transmitidas por alimentos, es un problema para la salud pública. Genera constantemente preocupación para detectar, reducir y monitorear la transmisión de los patógenos en la cadena de producción y procesamiento. Se demostró cómo se encontraron bajos niveles de calidad sanitaria e inocuidad alimentaria de cárnicos de tres provincias de la Región Amazonas (Bagua, Chachapoyas y Utcubamba). Posteriormente se realizaron capacitaciones en los diferentes puntos de la cadena de producción. Finalmente, los parámetros, físicos, químicos y microbiológicos se evaluaron con un análisis multivariado, demostrando una mejora significativa en la calidad del producto a nivel vendedor.

Palabras clave: inocuidad, físicos, químicos, microbiológicos, análisis multivariado.

\section{ABSTRACT}

Meat, as a source of foodborne disease, is a public health problem. It constantly generates concern for detecting, reducing, and monitoring the transmission of pathogens in the production and processing chain. It was demonstrated how low levels of sanitary quality and food safety were found in meat from three provinces of the Amazon Region (Bagua, Chachapoyas, and Utcubamba). Subsequently, training was provided at different points in the production chain. Finally, the physical, chemical, and microbiological parameters were evaluated with multivariate analysis, demonstrating a significant improvement in the quality of the product at the seller's level.

Keywords: safety, physical, chemical, microbiological, multivariate analysis.

\footnotetext{
Universidad de la Frontera, Sullana, Perú

"Autor de Correspondencia, e-mail jhony.gonzales@unf.edu.pe
} 


\section{INTRODUCCIÓN}

Los sistemas de control de producción y las pruebas de autenticidad de alimentos están en creciente demanda, ya que representan los temas de interés tanto de consumidores como de productores y comercializadores en el mercado actual. Los sistemas de producción y los productos alimenticios pueden ser descritos como sistemas complejos, en donde varios factores pueden interactuar y jugar un papel fundamental (Walsh y Leva, 2019). Consecuentemente, todos estos factores deben ser monitoreados y controlados. El uso de técnicas de estadística multivariada para el control de procesos y calidad en la industria agroalimentaria ha tenido un crecimiento significativo desde la mitad de los años setenta. Facilita el análisis de matrices de datos de gran tamaño que pueden ser estudiadas simultáneamente y comprendidas de una manera rápida, eficiente y sencilla para resolver problemas extremadamente complejos (Zuluaga, 2011).

Las operaciones de la unidad, modifican las propiedades del material con el objetivo de producir productos alimenticios uniformes y de alta calidad. Estos deben contar con una mayor aceptación por parte de los consumidores, cada vez más exigentes, o con una vida útil más larga y mejores posibilidades de almacenamiento y transporte (Shukesheva et al., 2018).

Los microorganismos pueden aparecer en las diferentes operaciones unitarias, empleadas durante el procesamiento de alimentos. En la granja y en la elaboración las operaciones unitarias su presencia se da comúnmente en toda la cadena de producción de alimentos. La intensidad y combinación de las operaciones unitarias junto con la composición de los alimentos, el envasado y las condiciones de almacenamiento influirán en el predominio de microorganismos específicos, que pueden ser patógenos o responsables de la descomposición (Pina-Pérez et al., 2017).

Por lo tanto, la inocuidad de los alimentos, el conocimiento y la cuantificación de los efectos causados por cada etapa del procesamiento pueden permitir controlar y garantizar la calidad y la seguridad de los productos manufacturados (Alvarenga et al., 2018).
Es por esto, que el objetivo de esta investigación fue evaluar los parámetros físicos, químicos y microbiológicos de los productos cárnicos comercializados en la región Amazonas, a nivel de vendedor.

\section{MATERIALY MÉTODOS}

\section{Área de Estudio}

La ejecución de esta investigación se desarrolló en tres provincias de la Región Amazonas: Bagua, Chachapoyas y Utcubamba (Figura 1).

\section{Tamaño de muestra}

Los puntos de venta muestreados, situados en las diferentes provincias del Departamento Amazonas han sido determinados según la base de datos del Servicio Nacional de Sanidad Agraria (SENASA), y las entidades competentes por cada sector. Se realizó un muestreo a nivel de vendedor donde se recogieron 50 muestras por punto de venta y por tipo de carne. Al finalizar se obtuvieron 150 muestras de pollo, 150 muestras de carnes de cerdo y 150 muestras de carne de res.

\section{Muestras y técnicas}

El material biológico utilizado para realizar la evaluación fisicoquímica y microbiológica de 450 muestras de productos cárnicos, fueron los músculos de la región de la pierna en las diferentes especies. Se recolectaron $300 \mathrm{~g}$ de carne fresca en los centros de venta. Se colocaron de manera individual en bolsas ziploc identificadas con código único y trasladadas en cadena de frío al Laboratorio de Enfermedades Infecciosas y Parasitarias de Animales Domésticos de la UNTRM, donde se fraccionó, 50 g para análisis químico y 50 g para análisis físico en ambos tipos de muestras, mientras que para el análisis microbiológico se utilizaron $200 \mathrm{~g}$.

En el análisis físico se determinaron tres parámetros, la acidez titulable con el método de la volumetría, el $\mathrm{pH}$ utilizando un $\mathrm{pH}$-metro (marca OAKTON $\mathrm{Ph}$ 450), y la humedad colocando en la estufa (ECOCELL LSIS - B2V / EC a $60^{\circ} \mathrm{C}$ ) la muestra dentro de una placa Petri para luego aplicar el cálculo de porcentaje de humedad (M1 - M2) x 100/(M1 - M0).

Se analizaron cinco parámetros químicos, usando una estufa (ECOCELL LSIS - B2V / EC a $60^{\circ} \mathrm{C}$ ) para la 


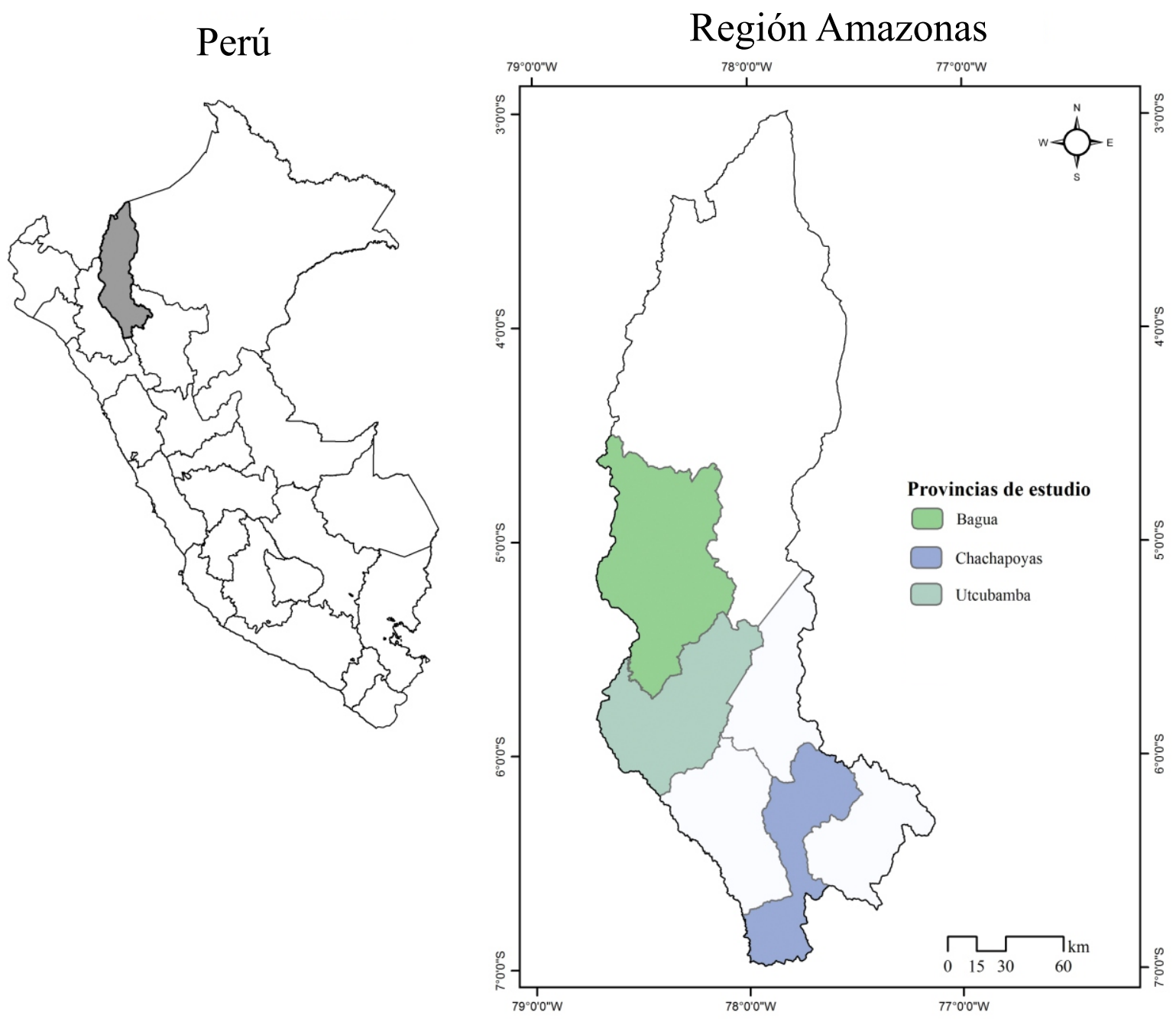

Figura 1. Mapa de ubicación del área de estudio en las provincias de la Región Amazonas.

determinación de materia seca, el método Soxhlet para determinación de grasa, una mufla para la determinación de cenizas, el método Kjeldhal para la determinación de proteína, y el método de combustión para la determinación de energía. En los análisis microbiológicos se determinaron coliformes totales y termotolerantes o fecales mediante la técnica del número más probable (NMP). Para detección de Salmonella sp, y enterobacterias se realizó mediante la técnica de siembra directa por estría en placa y para el conteo de bacterias aerobias mesófilas viables en placa se utilizó la técnica de siembra por incorporación. La determinación de histamina se realizó mediante ultra cromatografía líquida con el equipo UHPLC de marga Agilent Technologies modelo Infinity II 1290.

\section{Análisis de datos}

La técnica univariada que se aplicó fue el análisis estadístico t-student. Este permitió precisar evidencias acerca del comportamiento de los valores obtenidos en las muestras con respecto a los parámetros que establece la norma vigente, la NTS-071-MINSA/DIGESAV.01 (DIGESA, 2008).

La técnica multivariada que se utilizó para la caracterización fue el Análisis de Componentes Principales (ACP), que consiste en construir un conjunto de nuevas variables o componentes (incorrelacionadas), como combinación lineal de las variables originales, de forma tal que recojan toda la variabilidad existente. Esta técnica permite reducir la dimensionalidad del problema, por medio del estudio de las relaciones existentes entre las variables y el análisis de la disper- 
sión de las observaciones, y pone de evidencia posibles agrupamientos.

\section{RESULTADOS}

\section{Caracterización a nivel de vendedor vía univariada}

Los resultados de aplicación de la prueba de hipótesis se presentan en la Tabla 1. Es conveniente resaltar que los parámetros microbiológicos, aun cuando están por debajo de los límites máximos permisibles, se encuentran muy cercanos al nivel de significancia fijado para la investigación, que es de 0,05 (5\%), es decir, están muy cercanos al umbral de permisibilidad. Algunos de los parámetros microbiológicos podrían haber sido calificados como significativos o altamente significativos, si el nivel de significancia establecido fuese de $0,10(10 \%)$.

Tabla 1. Resultados prueba de hipótesis parámetros físicos, químicos y microbiológicos para cárnicos a nivel de vendedor

\begin{tabular}{|c|c|c|c|c|c|c|c|c|c|c|}
\hline \multirow{2}{*}{\multicolumn{2}{|c|}{ Parámetros }} & \multicolumn{3}{|c|}{ Bagua } & \multicolumn{3}{|c|}{ Chachapoyas } & \multicolumn{3}{|c|}{ Utcubamba } \\
\hline & & Pollo & Cerdo & Res & Pollo & Cerdo & Res & Pollo & Cerdo & Res \\
\hline \multirow{6}{*}{ Físicos } & \multirow{2}{*}{$\begin{array}{c}\text { Acidez } \\
\text { titulable }\end{array}$} & $\mathrm{t}: 1.69 \mathrm{~ns}$ & $\mathrm{t}: 1.73 \mathrm{~ns}$ & $\mathrm{t}: 1.77 \mathrm{~ns}$ & $\mathrm{t}: 1.99 \mathrm{~ns}$ & $\mathrm{t}: 1.54 \mathrm{~ns}$ & $\mathrm{t}: 1.81 \mathrm{~ns}$ & $\mathrm{t}: 1.83 \mathrm{~ns}$ & $\mathrm{t}: 1.90 \mathrm{~ns}$ & $\mathrm{t}: 1.64 \mathrm{~ns}$ \\
\hline & & $P: 0.593$ & $P: 0.385$ & $\mathrm{P}: 0.614$ & $\mathrm{P}: 0.354$ & $P: 0.651$ & P:0.393 & $\mathrm{P}: 0.313$ & $P: 0.308$ & P:0.417 \\
\hline & \multirow{2}{*}{ pH } & $\mathrm{t}: 1.87 \mathrm{~ns}$ & $\mathrm{t}: 1.82 \mathrm{~ns}$ & $\mathrm{t}: 1.78 \mathrm{~ns}$ & $\mathrm{t}: 1.88 \mathrm{~ns}$ & $\mathrm{t}: 1.76 \mathrm{~ns}$ & $\mathrm{t}: 1.90 \mathrm{~ns}$ & $\mathrm{t}: 1.66 \mathrm{~ns}$ & $\mathrm{t}: 1.82 \mathrm{~ns}$ & $\mathrm{t}: 1.52 \mathrm{~ns}$ \\
\hline & & $P: 0.663$ & $P: 0.493$ & $\mathrm{P}: 0.333$ & $\mathrm{P}: 0.441$ & $P: 0.341$ & P:0.387 & P:0.473 & $P: 0.342$ & P:0.455 \\
\hline & \multirow{2}{*}{ Humedad } & $\mathrm{t}: 1.53 \mathrm{~ns}$ & $\mathrm{t}: 1.33 \mathrm{~ns}$ & $\mathrm{t}: 1.69 \mathrm{~ns}$ & $\mathrm{t}: 1.76 \mathrm{~ns}$ & $\mathrm{t}: 1.65 \mathrm{~ns}$ & $\mathrm{t}: 1.59 \mathrm{~ns}$ & $\mathrm{t}: 1.73 \mathrm{~ns}$ & $\mathrm{t}: 1.57 \mathrm{~ns}$ & $\mathrm{t}: 1.32 \mathrm{~ns}$ \\
\hline & & $P: 0.502$ & $P: 0.357$ & $\mathrm{P}: 0.541$ & $\mathrm{P}: 0.494$ & $\mathrm{P}: 0.391$ & P:0.463 & P:0.411 & $\mathrm{P}: 0.402$ & $P: 0.567$ \\
\hline \multirow{10}{*}{ Químicos } & \multirow{2}{*}{$\begin{array}{c}\text { Materia } \\
\text { seca }\end{array}$} & $\mathrm{t}: 1.35 \mathrm{~ns}$ & $\mathrm{t}: 1.57 \mathrm{~ns}$ & $\mathrm{t}: 1.96 \mathrm{~ns}$ & $\mathrm{t}: 1.94 \mathrm{~ns}$ & $\mathrm{t}: 1.43 \mathrm{~ns}$ & $\mathrm{t}: 1.94 \mathrm{~ns}$ & $\mathrm{t}: 1.03 \mathrm{~ns}$ & $\mathrm{t}: 1.84 \mathrm{~ns}$ & $\mathrm{t}: 1.51 \mathrm{~ns}$ \\
\hline & & $P: 0.621$ & $P: 0.336$ & $\mathrm{P}: 0.314$ & $\mathrm{P}: 0.330$ & $P: 0.547$ & P:0.316 & $\mathrm{P}: 0.711$ & $\mathrm{P}: 0.311$ & P:0.440 \\
\hline & \multirow{2}{*}{ Grasa } & $\mathrm{t}: 1.78 \mathrm{~ns}$ & $\mathrm{t}: 1.97 \mathrm{~ns}$ & $\mathrm{t}: 1.97 \mathrm{~ns}$ & $\mathrm{t}: 1.88 \mathrm{~ns}$ & $\mathrm{t}: 1.62 \mathrm{~ns}$ & $\mathrm{t}: 1.71 \mathrm{~ns}$ & $\mathrm{t}: 1.95 \mathrm{~ns}$ & $\mathrm{t}: 1.76 \mathrm{~ns}$ & $\mathrm{t}: 1.55 \mathrm{~ns}$ \\
\hline & & $P: 0.431$ & $P: 0.645$ & $\mathrm{P}: 0.334$ & $\mathrm{P}: 0.348$ & $P: 0.531$ & P:0.423 & P:0.399 & P:0.399 & P:0.581 \\
\hline & \multirow{2}{*}{ Ceniza } & $\mathrm{t}: 1.66 \mathrm{~ns}$ & $\mathrm{t}: 1.55 \mathrm{~ns}$ & $\mathrm{t}: 1.97 \mathrm{~ns}$ & $\mathrm{t}: 1.79 \mathrm{~ns}$ & $\mathrm{t}: 1.65 \mathrm{~ns}$ & $\mathrm{t}: 1.73 \mathrm{~ns}$ & $\mathrm{t}: 1.94 \mathrm{~ns}$ & $\mathrm{t}: 1.81 \mathrm{~ns}$ & $\mathrm{t}: 1.74 \mathrm{~ns}$ \\
\hline & & $P: 0.413$ & $\mathrm{P}: 0.378$ & $\mathrm{P}: 0.314$ & $\mathrm{P}: 0.364$ & $P: 0.341$ & P:0.401 & $\mathrm{P}: 0.377$ & $P: 0.308$ & P:0.369 \\
\hline & \multirow{2}{*}{ Proteína } & $\mathrm{t}: 1.87 \mathrm{~ns}$ & $\mathrm{t}: 1.79 \mathrm{~ns}$ & $\mathrm{t}: 1.81 \mathrm{~ns}$ & $\mathrm{t}: 1.71 \mathrm{~ns}$ & $\mathrm{t}: 1.73 \mathrm{~ns}$ & $\mathrm{t}: 1.74 \mathrm{~ns}$ & $\mathrm{t}: 1.75 \mathrm{~ns}$ & $\mathrm{t}: 1.90 \mathrm{~ns}$ & $\mathrm{t}: 1.57 \mathrm{~ns}$ \\
\hline & & P:0.438 & $P: 0.361$ & $\mathrm{P}: 0.301$ & $\mathrm{P}: 0.420$ & $P: 0.432$ & P:0.438 & $\mathrm{P}: 0.400$ & P:0.309 & $P: 0.622$ \\
\hline & \multirow{2}{*}{ Energía } & $\mathrm{t}: 1.73 \mathrm{~ns}$ & $\mathrm{t}: 1.76 \mathrm{~ns}$ & $\mathrm{t}: 1.59 \mathrm{~ns}$ & $\mathrm{t}: 1.68 \mathrm{~ns}$ & $\mathrm{t}: 1.49 \mathrm{~ns}$ & $\mathrm{t}: 1.77 \mathrm{~ns}$ & $\mathrm{t}: 1.63 \mathrm{~ns}$ & $\mathrm{t}: 1.93 \mathrm{~ns}$ & $\mathrm{t}: 1.83 \mathrm{~ns}$ \\
\hline & & $P: 0.467$ & $\mathrm{P}: 0.443$ & $\mathrm{P}: 0.444$ & $\mathrm{P}: 0.424$ & $P: 0.667$ & P:0.346 & $P: 0.469$ & $\mathrm{P}: 0.349$ & $\mathrm{P}: 0.458$ \\
\hline \multirow{10}{*}{ Microbiológicos } & \multirow{6}{*}{$\begin{array}{c}\text { Coliformes } \\
\text { totales } \\
\text { Coliformes } \\
\text { fecales } \\
\text { Entero } \\
\text { bacterias }\end{array}$} & $\mathrm{t}: 2.38 \mathrm{~ns}$ & $\mathrm{t}: 2.49 \mathrm{~ns}$ & $\mathrm{t}: 2.37 \mathrm{~ns}$ & $\mathrm{t}: 2.40 \mathrm{~ns}$ & $\mathrm{t}: 2.31 \mathrm{~ns}$ & $\mathrm{t}: 2.15 \mathrm{~ns}$ & $\mathrm{t}: 2.19 \mathrm{~ns}$ & $\mathrm{t}: 2.38 \mathrm{~ns}$ & $\mathrm{t}: 2.46 \mathrm{~ns}$ \\
\hline & & $\mathrm{P}: 0.083$ & P:0.097 & $\mathrm{P}: 0.061$ & P:0.087 & P:0.094 & P:0.092 & P:0.099 & $\mathrm{P}: 0.091$ & $\mathrm{P}: 0.067$ \\
\hline & & $\mathrm{t}: 2.21 \mathrm{~ns}$ & $\mathrm{t}: 1.93 \mathrm{~ns}$ & $\mathrm{t}: 2.08 \mathrm{~ns}$ & $\mathrm{t}: 1.91 \mathrm{~ns}$ & $\mathrm{t}: 2.07 \mathrm{~ns}$ & $\mathrm{t}: 2.28 \mathrm{~ns}$ & $\mathrm{t}: 2.26 \mathrm{~ns}$ & $\mathrm{t}: 1.98 \mathrm{~ns}$ & $\mathrm{t}: 2.33 \mathrm{~ns}$ \\
\hline & & $P: 0.094$ & $P: 0.187$ & P:0.104 & $\mathrm{P}: 0.225$ & $P: 0.096$ & P:0.075 & $\mathrm{P}: 0.071$ & $P: 0.104$ & $P: 0062$ \\
\hline & & $\mathrm{t}: 1.98 \mathrm{~ns}$ & $\mathrm{t}: 2.03 \mathrm{~ns}$ & $\mathrm{t}: 1.95 \mathrm{~ns}$ & $\mathrm{t}: 1.87 \mathrm{~ns}$ & $\mathrm{t}: 1.87 \mathrm{~ns}$ & $\mathrm{t}: 1.89 \mathrm{~ns}$ & $\mathrm{t}: 1.80 \mathrm{~s}$ & $\mathrm{t}: 1.97 \mathrm{~ns}$ & $\mathrm{t}: 1.66 \mathrm{~ns}$ \\
\hline & & $P: 0.193$ & P:0.099 & $\mathrm{P}: 0.213$ & $\mathrm{P}: 0.227$ & $P: 0.234$ & $\mathrm{P}: 0.354$ & P:0.392 & $\mathrm{P}: 0.311$ & $P: 0.368$ \\
\hline & \multirow{2}{*}{ Salmonella } & $\mathrm{t}: 2.07 \mathrm{~ns}$ & $\mathrm{t}: 2.15 \mathrm{~ns}$ & $\mathrm{t}: 1.96 \mathrm{~ns}$ & $\mathrm{t}: 1.90 \mathrm{~ns}$ & $\mathrm{t}: 1.89 \mathrm{~ns}$ & $\mathrm{t}: 1.93 \mathrm{~ns}$ & $\mathrm{t}: 1.94 \mathrm{~ns}$ & $\mathrm{t}: 1.97 \mathrm{~ns}$ & $\mathrm{t}: 1.96 \mathrm{~ns}$ \\
\hline & & $P: 0.213$ & P:0.195 & $\mathrm{P}: 0.214$ & $\mathrm{P}: 0.301$ & $\mathrm{P}: 0.334$ & P:0.388 & $\mathrm{P}: 0.349$ & $P: 0.314$ & P:0.227 \\
\hline & \multirow{2}{*}{$\begin{array}{l}\text { Aérobicos } \\
\text { mesófilos }\end{array}$} & $\mathrm{t}: 2.11 \mathrm{~ns}$ & $\mathrm{t}: 2.17 \mathrm{~ns}$ & $\mathrm{t}: 2.14 \mathrm{~ns}$ & $\mathrm{t}: 1.94 \mathrm{~ns}$ & $\mathrm{t}: 1.97 \mathrm{~ns}$ & $\mathrm{t}: 2.31 \mathrm{~ns}$ & $\mathrm{t}: 2.07 \mathrm{~ns}$ & $\mathrm{t}: 2.06 \mathrm{~ns}$ & $\mathrm{t}: 2.26 \mathrm{~ns}$ \\
\hline & & P:0.093 & $P: 0.095$ & $\mathrm{P}: 0.066$ & $\mathrm{P}: 0.210$ & $P: 0.087$ & P:0.093 & $\mathrm{P}: 0.073$ & $\mathrm{P}: 0.090$ & $\mathrm{P}: 0.061$ \\
\hline \multirow{2}{*}{\multicolumn{2}{|c|}{ Histamina }} & $\mathrm{t}: 1.79 \mathrm{~ns}$ & $\mathrm{t}: 1.84 \mathrm{~ns}$ & $\mathrm{t}: 1.94 \mathrm{~ns}$ & $\mathrm{t}: 1.92 \mathrm{~ns}$ & $\mathrm{t}: 1.87 \mathrm{~ns}$ & $\mathrm{t}: 1.48 \mathrm{~ns}$ & $\mathrm{t}: 2.05 \mathrm{~ns}$ & $\mathrm{t}: 1.67 \mathrm{~ns}$ & $\mathrm{t}: 2.24 \mathrm{~ns}$ \\
\hline & & $P: 0.443$ & $\mathrm{P}: 0.314$ & P:0.337 & $\mathrm{P}: 0.334$ & $P: 0.398$ & P:0.688 & P:0.099 & $\mathrm{P}: 0.401$ & P:0.094 \\
\hline
\end{tabular}

(ns: No significativo)

\section{Caracterización a nivel de vendedor vía multivariada}

Los resultados obtenidos una vez aplicado el ACP se muestran en la tabla 2 y 3 . Donde la comunalidad es el porcentaje de la variabilidad que las componentes extraen de la variable, es la suma de los cuadrados de las correlaciones de las variables con los componentes. La alta comunalidad es el alto porcentaje de variabilidad de la variable explicada por los ejes considerados, es decir, es el "alejamiento" del centro en dirección a una o ambas componentes. En este caso, las dos componentes o factores explican una variabilidad del $66.5 \%$, que indica un buen nivel de explicación para dos factores. En tal sentido, se procedió a graficar los valores propios, las variables (parámetros físicos, quí-micos y microbio- lógicos) y los individuos (muestras), con el propósito de analizar el comportamiento del fenómeno en estudio, esto es, las similitudes, asociaciones y tendencias.

Las variables (parámetros) en el orden en que fueron procesadas, de acuerdo a la construcción de la matriz de información, presentan una tendencia a un comportamiento exponencial negativo, es decir, a medida que se van midiendo las variables, la respuesta obtenida va decreciendo (Figura 2).

Hay una fuerte asociación entre los parámetros materia seca, grasa y energía, y estos parámetros no tienen asociación alguna con el pH (Figura 3), aspecto que se corrobora con la matriz de correlaciones entre las componentes (Tabla 2). 
Tabla 2. Componentes principales del estudio sin rotar

\begin{tabular}{cccc}
\hline Variable & CP1 & CP2 & Comunalidad \\
\hline Coliformes fecales & $-0,236$ & $-0,273$ & 0,130 \\
Salmonella & 0,149 & $-0,151$ & 0,045 \\
Aerobios mesófilos & $-0,251$ & 0,163 & 0,090 \\
Materia seca & 0,715 & $-0,620$ & 0,895 \\
Ceniza & 0,285 & $-0,375$ & 0,222 \\
Grasa & 0,812 & 0,129 & 0,677 \\
Proteína & 0,187 & 0,151 & 0,058 \\
Energía & 0,716 & $-0,113$ & 0,525 \\
pH & $-0,517$ & $-0,693$ & 0,747 \\
Acidez & 0,636 & 0,289 & 0,488 \\
Humedad & $-0,715$ & 0,620 & 0,895 \\
Histamina & 0,593 & 0,671 & 0,802 \\
\hline Variancia & 3,4746 & 5,5748 \\
\%o Var & 0,390 & 0,275 & 0,665 \\
\hline
\end{tabular}

Tabla 3. Coeficientes de puntuación de los componentes principales del estudio

\begin{tabular}{ccc}
\hline Variable & CP1 & CP2 \\
\hline Coliformes fecales & $-0,068$ & $-0,130$ \\
Salmonella & 0,043 & $-0,072$ \\
Aerobios mesófilos & $-0,072$ & 0,078 \\
Materia seca & 0,411 & $-0,590$ \\
Ceniza & 0,082 & $-0,178$ \\
Grasa & 0,234 & 0,062 \\
Proteína & 0,054 & 0,072 \\
Energía & 0,206 & $-0,054$ \\
pH & $-0,149$ & $-0,330$ \\
Acidez & 0,183 & 0,138 \\
Humedad & $-0,000$ & $-0,000$ \\
Histamina & 0,171 & 0,320 \\
\hline
\end{tabular}

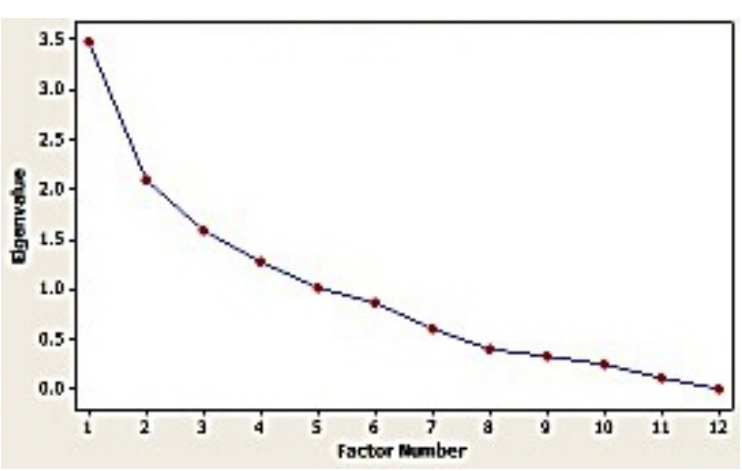

Figura 2. Valores propios del ACP.

\section{IV.DISCUSIÓN}

La calidad sanitaria e inocuidad alimentaria de cárnicos son sumamente importantes para la salud pública, además de imponer la competitividad de estos productos en el mercado. Por lo que, los resultados de esta investigación demuestron que es necesario realizar el monitoreo constante a lo largo de la cadena de producción obteniendo de los parámetros físicos, químicos y

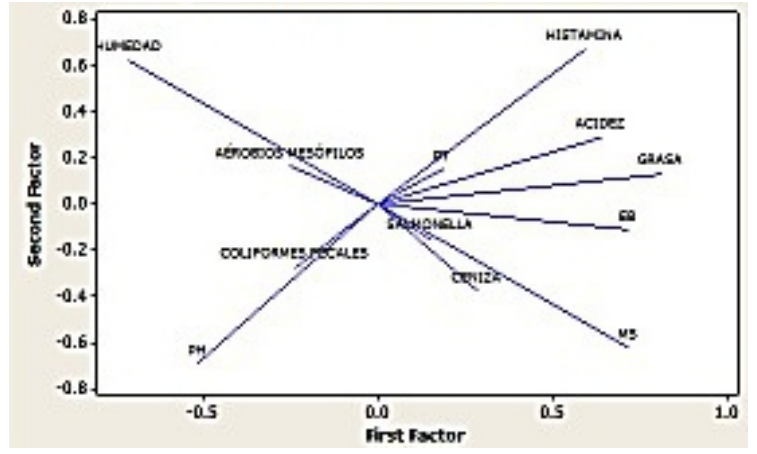

Figura 3. Gráfica de ACP que describe el comportamiento de las variables estudiadas.

microbiológicos. Weinroth et al., 2018, de igual manera menciona que la detección, reducción y monitoreo de la salud pública de patógenos transmitidos por los alimentos ha avanzado en precisión y eficiencia a medida que la tecnología ha progresado afectando la gestión de la seguridad alimentaria y la salud pública en el futuro. Fegan y Jenson (2018), corroboran que la carne es fuente de enfermedades y puede detectarse en varios puntos de la cadena de suministro, determinar la fuente de dónde surgen estos durante la producción y el procesamiento, junto con el análisis de datos para investigar la atribución de fuentes y el comportamiento de los patógenos han permitido comprender mejor cómo se producen los brotes de transmisión alimentaria y dónde se pueden implementar los controles. Por lo que se hace necesario la intervención de políticas de estado que implementen sistemas de trazabilidad donde sea posible seguir el rastro de toda la cadena de producción para tomar las medidas adecuadas y brin- 
dar la alimentación adecuada a la población.

Los análisis demuestran que no existe asociación de la materia seca con el $\mathrm{pH}$, pero este influye en la proliferación de microorganismos, por lo que el $\mathrm{pH}$ de la carne debe oscilar entre 5,5 y 6,4 (NTP, 2008). De la misma forma los microorganismos establecen mecanismos de respuesta para lidiar con el estrés, lo que les permite sobrevivir en determinadas condiciones de $\mathrm{pH}$ y proliferarse (Petruzzi et al., 2017).

\section{CONCLUSIÓN}

Se comprobó positivamente que los puntos de venta estudiados cumplen en las características físicas, químicas y microbiológicas de los productos cárnicos que se expenden en la región Amazonas. Sin embargo, hay que seguir desarrollando estrategias de concientización para lograr la mejora en términos de calidad sanitaria e inocuidad alimentaria, ya que los aspectos microbiológicos deben ser afianzados y consolidados, y para ello hay que impulsar un plan permanente de formación, capacitación, monitoreo y supervisión de la comercialización y distribución de productos cárnicos para lograr que los productos lleguen al consumidor de la mejor forma posible y esto exige estrategias para el control de procesos y la gestión de la calidad.

\section{REFERENCIAS BIBLIOGRÁFICAS}

Alvarenga V., F. Campagnollo, L. Prado, C. Horita, M. Caturla, E. Pereira, A. Crucello y A. Sant'Ana. 2018. "Impact of Unit Operations From Farm to Fork on Microbial Safety and Quality of Foods." Advances in Food and Nutrition Research. 85: 131-175. DOI: 10.1016/bs.afnr.2018.02.004.

Fegan N., y I. Jenson. 2018. "The role of meat in foodborne disease: Is there a coming revolution in risk assessment and management."Meat Science 144: 22-29. DOI: 10.1016/j.meatsci.2018.04.018.

DIGESA (Dirección General de Salud Ambiental). 2011. "Procedimiento para la Recepción de Muestras de Alimentos y Bebidas de Consumo Humano en el Laboratorio de Control Ambien- tal de la Dirección General de Salud Ambiental del Ministerio de Salud." Lima (Perú).

DIGESA (Dirección General de Salud Ambiental). 2008. "Norma Sanitaria que establece los criterios microbiológicos de calidad sanitaria e inocuidad para los alimentos y bebidas de consumo humano.” Lima (Perú).

NTP (Norma Técnica Peruana). 2008. "NTP 201.005:2008. Carne y productos cárnicos. Definiciones, clasificación y requisitos de carcasas y carnes de bovinos.” Lima (Perú)

Pina-Pérez, M. C., A. Rivas, A. Martínez, y D. Rodrigo. 2017. "Antimicrobial potential of macro and microalgae against pathogenic and spoilage microorganisms in food." Food Chemis$t r y \quad 235: 34-44$. D O I : 10.1016/j.foodchem.2017.05.033

Petruzzi L., M. Corb, M. Sinigaglia, y A. Bevilacqua. 2017. "Chapter 1 - Microbial Spoilage of Foods: Fundamentals." The Microbiological Quality of Food 1-21. DOI: 10.1016/B978-008-100502-6.00002-9

Shukesheva S. E., Y. M. Uzakov, I. M. Chernukha, D. E. Nurmukhanbetova, Z. S. Nabiyeva, y A. B. Nurtaeva. 2018. "Research to improve the quality of food products." News of the National Academy of Sciences of the Republic of Kazakhstan, Series of Geology and Technical Sciences 3 (430): 37-45.

Walsh, C. y M. C. Leva. 2019. "A review of human factors and food safety in Ireland." Safety Science 119:399-411. DO I : 10.1016/j.ssci.2018.07.022

Weinroth M., B. Britton, y K. Belk. 2018. "Genetics and microbiology of meat." Meat Science 144: 15-21. DOI: 10.1016/j.meatsci.2018.04.018.

Zuluaga, C. M. 2011. “Análisis estadístico multivariado: una herramienta estratégica para el control de procesos y calidad en la industria agroalimentaria." Revista especializada en ingeniería de procesos en alimentos biomateriales UNAD. (5): 143-157. 